\title{
Small, Low-energy, Dispersive Solar Energetic Particle Events Observed by Parker Solar Probe
}

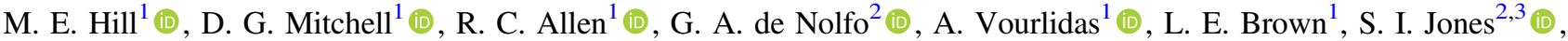 \\ D. J. McComas ${ }^{4}\left(10\right.$, R. L. McNutt, Jr. ${ }^{1}$, J. G. Mitchell ${ }^{2}$ (i), J. R. Szalay ${ }^{4}$ (i), S. Wallace ${ }^{2}$ (1), C. N. Arge ${ }^{2}$, E. R. Christian ${ }^{2}$ (1),

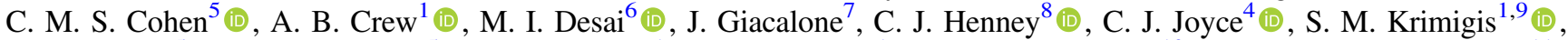

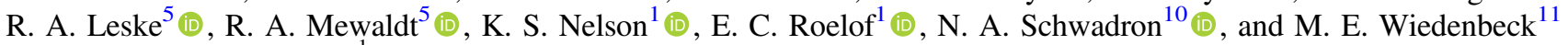 \\ 1 Johns Hopkins University Applied Physics Laboratory, Laurel, MD 20742, USA \\ ${ }^{2}$ Solar Physics Laboratory, NASA Goddard Spaceflight Center, Greenbelt, MD 20771, USA \\ ${ }^{3}$ Catholic University of America, Washington, DC 20064, USA \\ ${ }^{4}$ Department of Astrophysical Science, Princeton University, Princeton, NJ 08544, USA \\ ${ }^{5}$ California Institute of Technology, Pasadena, CA 91125, USA \\ ${ }^{6}$ Southwest Research Institute, San Antonio, TX 78228, USA \\ ${ }^{7}$ University of Arizona, Tucson, AZ 85721, USA \\ ${ }^{8}$ Air Force Research Laboratory, Space Vehicles Directorate, Kirtland AFB, NM 87117, USA \\ ${ }^{9}$ Office for Space Research and Technology, Academy of Athens, Athens, Greece \\ ${ }_{10}$ University of New Hampshire, Durham, NH 03824, USA \\ ${ }^{11}$ Jet Propulsion Laboratory, California Institute of Technology, Pasadena, CA 91109, USA \\ Received 2019 October 4; revised 2019 November 15; accepted 2019 November 17; published 2020 February 3
}

\begin{abstract}
The Energetic Particle Instrument-Low Energy (EPI-Lo) experiment has detected several weak, low-energy $\left(\sim 30-300 \mathrm{keV}\right.$ nucleon ${ }^{-1}$ ) solar energetic particle (SEP) events during its first two closest approaches to the Sun, providing a unique opportunity to explore the sources of low-energy particle acceleration. As part of the Parker Solar Probe (PSP) Integrated Science Investigation of the Sun (IS $\odot$ IS) suite, EPI-Lo was designed to investigate the physics of energetic particles; however, in the special lowest-energy "time-of-flight only" product used in this study, it also responds to solar photons in a subset of approximately sunward-looking apertures lacking special light-attenuating foils. During the first three perihelia, in a frame rotating with the Sun, PSP undergoes retrograde motion, covering a $17^{\circ}$ heliographic longitudinal range three times during the course of the $\sim 11$-day perihelion passes, permitting a unique spatial and temporal study into the location, correlation, and persistence of previously unmeasurable SEPs. We examine the signatures of these SEPs (during the first PSP perihelion pass only) and the connection to possible solar sources using remote observations from the Solar Dynamics Observatory (SDO), the Solar TErrestrial RElations Observatory (STEREO), and the ground-based Global Oscillation Network Group (GONG). The orientation of the Sun relative to STEREO, SDO, and GONG makes such identifications challenging, but we do have several candidates, including an equatorial coronal hole at a Carrington longitude of $\sim 335^{\circ}$. To analyze observations from EPI-Lo, which is a new type of particle instrument, we examine instrumental effects and provide a preliminary separation of the ion signal from the photon background.

Unified Astronomy Thesaurus concepts: The Sun (1693); Interplanetary particle acceleration (826); Solar energetic particles (1491); Space vehicle instruments (1548); Solar flares (1496); Michelson interferometers (1044); Solar extreme ultraviolet emission (1493); Solar filament eruptions (1981); Quiet sun (1322); Solar telescopes (1531); Solar coronal mass ejections (310); Space probes (1545)
\end{abstract}

\section{Introduction}

The Parker Solar Probe (PSP) mission (Kinnison et al. 2013; Fox et al. 2014) investigates the Sun over the course of $8 \mathrm{yr}$ and 24 orbits, shedding angular momentum during seven Venus flybys to bring the spacecraft increasingly closer to the Sun, with perihelia ranging from 0.17 to $0.05 \mathrm{au}$. To date, complete data from the first two orbits, with 0.17 au perihelia on 2018 November 6 (day of year (DOY) 310) and 2019 April 4 (DOY 094), have been downlinked, and the third orbit, with 0.17 au perihelion on 2019 September 1 (DOY 244), has been completed safely, and data are in the process of being downlinked. First-results reports from orbits 1 and 2 (Bale et al. 2019; Howard et al. 2019; Kasper et al. 2019; McComas et al. 2019) provide an overview of the two closest encounters with the Sun yet achieved. Herein, we restrict the PSP observations to the first perihelion (Figure 1).

Solar flares are prodigious producers of energetic particles and thus a rich laboratory for studying particle acceleration. The acceleration occurs through the release of magnetic energy, a significant fraction of which can go into the acceleration of particles (Benz 2008; Krucker et al. 2010; Fleishman et al. 2011). Acceleration within the flare occurs low in the corona, perhaps in a reconnection region (Masuda et al. 1994; Battaglia $\&$ Benz 2006). Coronal mass ejections (CMEs) produce shocks that both accelerate particles and provide a mechanism for escape into the interplanetary (IP) medium. In order to observe them at a given position at $1 \mathrm{au}$, the assumption is that accelerated electrons and ions from the flare or CME eventually reach open magnetic field lines extending out into the IP medium. Additionally, particles local to a CME are directly observable when the propagating CME and the observer's positions intersect.

Although the details of particle acceleration are still being debated, evidence for particle acceleration can be found in the $\mathrm{X}$-ray and $\gamma$-ray emission resulting from interactions of accelerated particles in the dense chromosphere. Particle acceleration is also evident in solar energetic particle (SEP) 

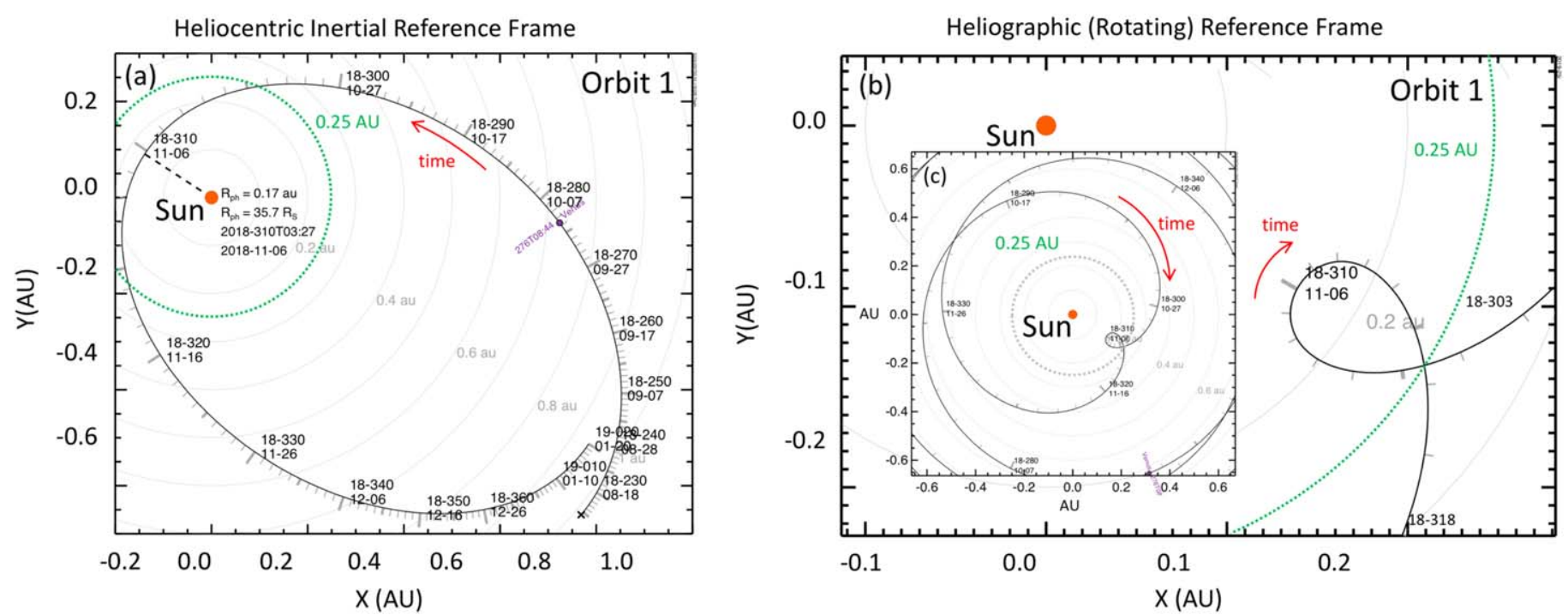

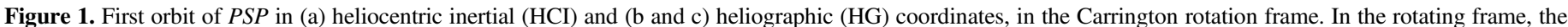

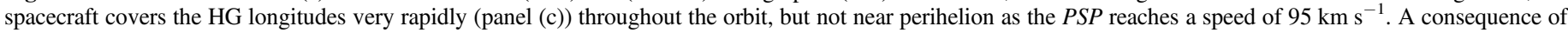

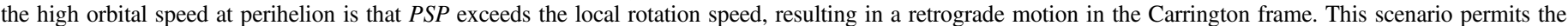
spacecraft to slowly scan a $17^{\circ}$ longitudinal range over the 11-day period, with each angle being visited three times during this period (panel (b)).

events. SEP events generally fall into two categories depending on their composition, spectra, and temporal behavior: gradual and impulsive events (Reames 1999). Admixtures of these two types of events are not uncommon (Cane et al. 2003; Li et al. 2007a, 2007b; Cohen et al. 2008). Generally speaking, gradual events are long-lasting and proton rich, having a composition reflecting a coronal origin. These particles are thought to be accelerated in CME-driven shocks high in the corona. Impulsive SEP events are typically thought to originate from magnetic reconnection lower in the corona, although the magnetic topology and acceleration processes are not well understood.

For flare-accelerated particles to escape, reconnection must take place between open and closed magnetic field lines, perhaps escaping from emerging magnetic flux near the boundaries of sunspots, which are thought to produce jet-like features (Shibatta et al. 1992; Shimojo et al. 1996, 1998). During reconnection, or as a result of reconnection, beams of electrons are produced that travel upward and downward along magnetic field lines in the corona. Downward-propagating electrons interact in the dense chromosphere to produce hard X-ray (HXR) emission. These downward accelerated electrons (up to $\sim 100 \mathrm{keV}$ ) contain $\sim 10 \%-50 \%$ of the total energy released in flares, suggesting that the energy release during flares is responsible for particle acceleration (Lin et al. 2003). Clear emission features (magnetic footpoint [FP] and coronal loop top thermal and nonthermal electron emission) have been observed, pointing to acceleration due to reconnection (Masuda et al. 1994; Lin et al. 2003; Krucker et al. 2008). As a consequence of these interactions, chromospheric plasma is heated to high temperatures and expands into the corona, where it is detected in extreme-ultraviolet (EUV) and/or soft X-ray (SXR) (Benz 2008).

From the point of view of the magnetic energy release, accelerated ions can be as important as the electrons, perhaps more so (Ramaty et al. 1995; Ramaty \& Mandzhavidze 2000). Few flares exhibit gamma-ray emission, because either the intensity is below instrumental sensitivity or the ion spectrum does not surpass the gamma-ray production threshold.
However, few doubt that ions are very commonly accelerated in flares. Because there has not been a clear signature of ion escape, as is the case with the type III radio emission for electrons, sorting out the avenues of escape for accelerated flare ions and the possible origin of the impulsive SEPs continues to be a major challenge. Impulsive SEPs have also been associated with EUV jets (Nitta et al. 2015 and references therein), but unambiguous solar source identification relies heavily on associations with electron events and other electron signatures (e.g., type III emission, impulsive HXR emission). In many cases, the impulsive event is associated with other eruptive phenomena (e.g., loop structures, EUV waves; see Bučík et al. 2018), and the magnetic topology for ion escape is even less clear (Nitta et al. 2015).

One of the main obstacles to studying energetic particles originating at remote sources near the Sun using observations at $1 \mathrm{au}$ is that transport effects can alter the observed intensity and energy spectra in ways that are not well understood. Ion velocity dispersion can be used but tends to produce injection times with uncertainties of $\sim 1-2 \mathrm{hr}$, mainly due to long travel times from the Sun to $1 \mathrm{au}(>6 \mathrm{hr})$. In many cases, impulsive events can be connected to a solar source through timing considerations, although the assumption is that electrons and ions travel scatter-free. Transport modeling may be needed when the scatter-free approximation does not hold. The Integrated Science Investigation of the Sun (IS $\odot$ IS; McComas et al. 2016) was developed in part to address this difficulty by exploring the energetic particle environment, including SEP activity, closer to the Sun. This has been accomplished using observations from the two IS $\odot$ IS instruments, the Energetic Particle Instrument-Low Energy and High Energy (EPI-Lo and EPI-Hi), to obtain wide energy coverage $\left(\sim 20 \mathrm{keV}_{\text {nucleon }}{ }^{-1}\right.$

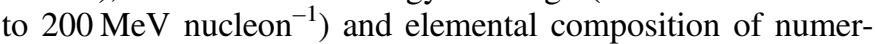
ous species, from $\mathrm{H}$ to Fe. Not only is IS $\odot$ IS able to sensitively detect impulsive events, many of which are too small to be observed from 1 au, but PSP's close proximity to the Sun could allow us to better identify the associated solar source and understand the acceleration mechanism. 
Wiedenbeck et al. (2020), Leske et al. (2020), and Cohen et al. (2020) have written reports concentrating on the EPI-Hi observations; Mitchell et al. (2020) and Giacalone et al. (2020) have focused on EPI-Lo measurements; Joyce et al. (2020) and Schwadron et al. (2020) have performed studies combining measurements from both IS $\odot$ IS instruments; and McComas et al. (2019) published the first PSP energetic particle results, providing an overview of all of the IS $\odot$ IS observations from the first two orbits. In this paper, we use the lowest-energy measurements from the IS $\odot$ IS suite to examine the small SEPs observed during the first $P S P$ perihelion pass. To achieve the lowest-energy measurements, we must use an EPI-Lo data product that is more prone to background than most of our measurements, which have more stringent detection logic; thus, we first discuss the instrument-specific analysis we have undertaken for separating foreground ions from the timedependent background, which is dominated by photons. Then, we discuss the SEP events themselves by analyzing their source timing and position estimates based on the EPI-Lo measurements and compare this to remote measurements from $1 \mathrm{au}$, seeking evidence for a connection between activity seen on the Sun and in situ SEPs detected by EPI-Lo.

\section{EPI-Lo Ion Measurements and the Effects of Photons}

The IS $\odot$ IS investigation's primary scientific goals laid out by McComas et al. (2016) are, briefly, to probe the dynamics of energetic particle origins, acceleration, and transport in the vicinity of the Sun. Both EPI-Lo and EPI-Hi have been returning particle measurements for the past $\sim 1$ yr. The IS $\odot$ IS / EPI-Lo instrument is the first (and, so far, only) flight model of the "Mushroom" (so named because of its domed shape, arising from the distinctive half-sky coverage, densely sampling $2 \pi \mathrm{sr}$ with 80 apertures). The EPI-Lo instrument design at the time of the PSP Preliminary Design Review is described by McComas et al. (2016), and a discussion of the general Mushroom measurement technique and its application (Hill et al. 2017), most of which is directly related to EPI-Lo, is also available. EPI-Lo is a time-of-flight (TOF) mass spectrometer composed of eight $45^{\circ}$ wedges (or octants), each with 10 apertures. These apertures use start and stop foils that, when penetrated by a primary ion, emit secondary electrons that are accelerated toward and amplified by a microchannel plate (MCP) paired with an arrangement of position-sensing start anodes. The primary ion continues until it strikes the stop foil, whose secondary electrons are accelerated, reflected, and detected with the same MCP/anode system. The result is a measurement of TOF and direction, therefore providing the particle velocity. Finally, below the stop foil, a solid-state detector (SSD) assembly of three SSDs located in the vertex of each wedge measures the deposited energy. The energy measurement combined with the velocity measurement permits a determination of the particle's mass, energy, and direction of travel. Note that although there are eight wedges, adjacent pairs are each serviced by one of four position-sensing anode systems, forming four largely independent quadrants, each controlled by a separate state machine in the field programmable gate array (FPGA) where the event logic, data processing, and many other functions occur.

The design is robust against the detrimental effects of ambient dust or light entering any of EPI-Lo's 80 apertures. The mitigation for light contamination includes employing thicker start foils in the six look directions dominated by photospheric light that is Thomson scattering off electrons near the Sun, and thus visible away from the solar disk where there is no shielding from PSP's thermal protection system (TPS). In addition to thicker foils, we employ baffles and multiple coincidence logic to cut down photon contamination. Also, to protect against dust and resulting dust-hole-admitted light, an extra "collimator foil" was added to all collimator turrets so that pinholes from dust impacts either penetrate only the collimator foil (for the smallest dust grains $\lesssim 100 \mathrm{~nm}$ ) or only admit light from very tiny solid angles where these holes line up, resulting in a more greatly reduced geometry factor for post-impact light contamination than would occur with only one foil. The first dust impact directly detectable by EPI-Lo (i.e., an impact resulting in noticeable light-admitting damage) took place after the second perihelion on 2019 April 3 (DOY 093) 16:45 in the L31 direction (see Figure 2 for description of EPI-Lo look directions). This L31 hole resulted in elevated photon background in one look direction but at a level that has not diminished EPI-Lo's scientific capabilities. This dust impact is discussed in more detail by Szalay et al. (2020).

In flight, we learned the extent to which solar photons are detectable by EPI-Lo even without a dust hole. The EPI-Lo foils were designed for average conditions. During flares, much harder UV and X-ray emission is typical, and at much higher intensity than the background solar flux. We could not design for those elevated transient levels, as it would have resulted in unacceptably high energy thresholds for the ions. It appears that when photons scatter off dust at sufficiently long elongation angles, it permits the light to illuminate the instrument's higher-elevation apertures, which have thinner start foils that are less able to attenuate the light. The transmitted light results in secondary electrons that are accelerated inside the sensor and detected just as they would be if they were produced by the intended incident ions, therefore becoming detectable when the photon intensities are high enough for accidental coincidences to occur. Accidental coincidence events preferentially trigger measurements with low energies and a distinct spectral shape (see Section 3.2). These photons, when they reach high intensities, can also produce enough events for the start pulse to cross-talk to the stop anode, producing coincidences at preferentially short TOFs (because the induced signal during a cross-talk event follows the primary signal rapidly) — thus mimicking the TOF of high-energy ions. We use our understanding of the way photon background is distributed in apparent energy, along with the 80 look directions to separate ion events from photon events (Section 3.1).

To distinguish between photons and ions, we need to distinguish the types of particles that EPI-Lo can detect. Here we present and discuss TOF-only measurements exclusively. Understanding the TOF-only measurement modalities (as distinct from the instrument modes mentioned below in Section 3.1) requires knowledge of (1) the type of events that can trigger EPI-Lo, (2) the detector subsystem triggered by each triggering event, and (3) how the various triggers combine to satisfy valid event logic. Table 1 summarizes much of this information. The measurement triggers are galactic cosmic-ray (GCR) starts and stops (GCRs that penetrate the instrument housing and induce an avalanche of electrons in the MCP that are collected on 1 of the 10 start anodes or single stop anodes per wedge), ion starts and stops (start or stop signals produced by foreground ions but for which, because of the expected 


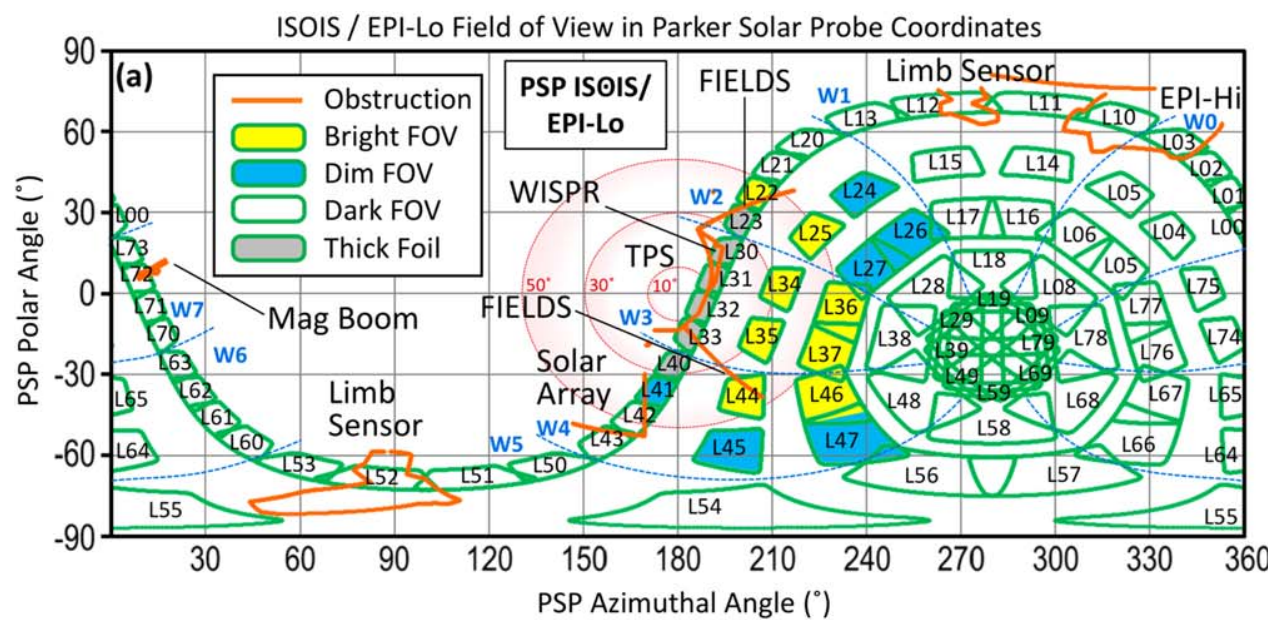

ISOIS/EPI-Lo Look Directions (Instrument Frame)

gure 2. (a) IS $\odot$ IS $/$ EPI-Lo FOV in $P S P$ spacecraft coordinates, where the coordinate $\left(180^{\circ}, 0^{\circ}\right)$ marks the location of the Sun during nominal perihelion pointing and the perihelion ram direction is located at $\left(270^{\circ}, 0^{\circ}\right)$. Green shapes indicate EPI-Lo's aperture FOVs, with the "bright" and "dim" look directions described in the text indicated with yellow and blue, respectively. The white- and gray-filled shapes both represent apertures that are defined as "dark," but the gray-colored apertures have the special thicker foils, so in their case the light reduction is a result of the attenuating characteristics of the foil rather than look direction. Obstructions are indicated in orange, where the solar array is shown in perihelion configuration. Blue text labels the eight wedges, W0-W7, which are separated by dashed blue lines, and the 80 look directions, L00-L79, are similarly indicated, in black. Note that the H9 (hole-9) apertures (L09, L19, ..., L70) have heavily overlapping FOVs, which are difficult to unambiguously represent. Angular contours at $10^{\circ}, 30^{\circ}$, and $50^{\circ}$ are represented with pink concentric ovals. (b) Alternate representation of the EPI-Lo apertures, in the instrument frame. The wedges and look directions are labeled analogously to panel (a), but notice the opposite sense of the numbering, which is a result of either "looking down" on the instrument or "looking up" at the sky. Notice that the most sunward wedge, W3, is highlighted in red. This compact instrument format for representing sky images is utilized in Figure 3.

Table 1

EPI-Lo TOF-only ${ }^{\mathrm{a}}$ Foreground and Background Modalities ${ }^{\mathrm{b}, \mathrm{c}}$

\begin{tabular}{|c|c|c|c|c|c|}
\hline \multirow[b]{2}{*}{ Start Triggers } & \multirow[b]{2}{*}{ Ion } & \multicolumn{2}{|c|}{ Stop Triggers } & \multirow[b]{2}{*}{ Cross Talk } & \multirow[b]{2}{*}{ GCR } \\
\hline & & Photon & Pulser & & \\
\hline Ion & $A, u^{d}$ & $b$ & C & $d$ & e \\
\hline Photon & $f$ & G & $\mathrm{h}$ & $i$ & j \\
\hline Pulser & $\mathbf{k}$ & 1 & $\mathbf{M}$ & $\mathrm{n}$ & 0 \\
\hline GCR & $\mathrm{p}$ & $q$ & $r$ & $\mathbf{S}$ & $t$ \\
\hline
\end{tabular}

Notes.

${ }^{a}$ The pulser also includes an SSD pulse, but it is included here since it impacts the start and stop rates.

${ }^{\mathrm{b}}$ Letters (alphabetical order) label the coincidences, which contribute to the proxy grid in Table 2 .

${ }^{\mathrm{c}}$ Capitalized labels highlight intended foreground ion (A) and pulser (M) signals, along with the chief background contribution, photon-photon accidentals (G), and GCR cross talk (S).

${ }^{\mathrm{d}} \mathbf{A}$ corresponds to ion foreground, and $\mathbf{u}$ corresponds to accidentals between uncorrelated ion starts and ion stops.

$<100 \%$ detection efficiency, two coincident signals were not produced), and photon start and stop (a photon that is transmitted through the foil, resulting in the emission of a secondary electron that is then detected by the normal means, or a transmitted photon that hits the stop foil, producing a stop pulse). In addition to these triggers, there can be a stop pulse that is induced (due to, for example, capacitive coupling) by a start pulse on the anode board, which we will label as cross talk. Start pulses induced by stop pulses also occur but are filtered out by the event logic, which requires that the start precede the stop in time. Finally, EPI-Lo uses a $1 \mathrm{kHz}$ per quadrant pulser to calculate the instrument live time, but although these pulser events are tagged by a special position measurement (position 20 in each quadrant), this position can be shifted to a real position (positions 0-19 in each quadrant), losing the pulser identification when there is a nonpulser signal in close proximity to the pulser event.
In Table 1 the measurement triggers for starts (ion, photon, pulser, and GCR) and stops (ion, photon, pulser, cross talk, and GCR) are shown with alphabetical labels, "a" to "u," keyed to Table 2. Capitalized labels are used to increase visibility of the intended foreground ion (A) and pulser (M) signals, along with the chief background contribution, photon-photon accidentals (G), and GCR cross talk (S). Table 2 shows the three-by-three grid used to define the ion and photon proxies; below the grid, the events tagged as pulser are listed. The relative contribution of the different measurement modalities is indicated with a tilde when contributions are comparable (during a notional combined ion and photon event) and inequalities elsewise. For example, the dark, low-energy cell contains the designation A > tep, which means that the A modality (foreground ions) contributes more to this set of energy channels and look directions than the GCR-GCR (modality $t$ ), ion-GCR (modality e), and GCR-ion (modality $\mathbf{p}$ ) background modalities. The constituent channels for the ion and photon proxies are highlighted by braces and brackets, respectively (and defined explicitly in Section 3.1).

\section{Analysis}

To properly analyze these small, low-energy SEPs we have to remove background. When exposed to more intense foreground events, the background signals are of less importance. One method to account for background is to examine the science measurements in detail and identify based on the energy and angular distribution which signals are consistent with a foreground source and which are responding most strongly to background. In addition to science measurements, EPI-Lo returns instrumental and engineering measurements; here we concentrate on what are termed "basic rates" (for this study we are employing the rates known as Ion Composition Basic Rates, ICB, which are associated only with the [T] science data product; see Table 3). We can use these basic rates (for which fewer logical requirements are imposed) 
Table 2

Ion and Photon Proxy Grid ${ }^{\mathrm{a}, \mathrm{b}}$

\begin{tabular}{|c|c|c|c|}
\hline \multirow{2}{*}{ Look Directions ${ }^{c}$} & \multicolumn{3}{|c|}{ Energy Ranges $^{c}$} \\
\hline & Low Energy & Medium Energy & High Energy \\
\hline $\begin{array}{l}\text { Bright } \\
\text { Dim } \\
\text { Dark }\end{array}$ & $\begin{array}{c}{[A \sim G>b q j f>\text { tep }]^{d}} \\
A>G>b q j f>\text { tep } \\
\{A>\text { tep }\}^{g}\end{array}$ & $\begin{array}{c}A>G>h^{\prime} \sim \mathbf{c}^{\prime}>\mathbf{r}^{\prime^{e}} \\
\left\{A>C^{\prime}>h^{\prime}>r^{\prime}\right\}^{e, g} \\
\left\{A>c^{\prime}>\mathbf{r}^{\prime}\right\}^{e, g}\end{array}$ & $\begin{aligned} d & \sim i \sim S^{f}>A \\
d & \sim S^{f}>A \\
d & \sim S^{f}>A\end{aligned}$ \\
\hline Pulser & $\ldots$ & $M^{\prime}>1^{\prime}{ }^{e}$ & $1^{\prime} \sim \mathrm{k}^{\prime}>\mathrm{n}^{\prime}>0^{\prime}$ \\
\hline
\end{tabular}

Notes.

${ }^{\text {a }}$ Letters refer to modalities described in Table 1.

b "Great than" symbols "> indicate the typical order in which modalities contribute to the measurements, where tildes " " indicate a comparable contribution during a notional combined ion and photon event.

${ }^{c}$ Look direction groupings and energy ranges are defined in Section 3.1. See also Figure 2.

${ }^{\mathrm{d}}$ Brackets highlight the data contributing to the photon proxy.

e Primes indicate that these events are triple coincidences with TOF and SSD energy.

${ }^{\mathrm{f}}$ During quiet times, the GCR cross-talk (S) contribution can dominate (see the "green" $\sim 1-10 \mathrm{MeV}$ nucleon ${ }^{-1}$ feature in Figure 3(a)).

${ }^{\mathrm{g}}$ Braces highlight the data contribution to the ion proxy.

to quantitatively disentangle the foreground and background contributions to the science measurements. The IS $\odot$ IS data used herein are available at http://spp-isois.sr.unh.edu/data_ public/, as well as the NASA Space Physics Data Facility, as described in Table 3 . The use of the TOF-only measurements is intended to lower the energy threshold of the instrument because the composition measurements begin at $\sim 100 \mathrm{keV}$ owing to the required detection in the SSD system and the associated energy loss before the ion reaches the active volume of the SSD. The trade-off is that background is more of a challenge than with the other higher-order coincidence logic data products. Here we present our initial effort to separate the intended ion signal from the photon background in this lowestenergy energetic particle measurement from PSP.

\subsection{Ion and Photon Proxies}

To produce proxies for ion and photon activity, we devised a three-by-three grid (Table 2) where each of the nine grid cells is composed of a set of channels of different energies and look directions (Figure 2 describes the look directions as projected on the sky and in the instrument frame). We divided the field of view (FOV) into three independent sets of look directions: the generally sunward-looking bright directions with very clear photon viewing (composed of the look directions L22, L25, L34-L37, L44, and L46); the dim directions surrounding the bright area, where there are reduced indications of photons (composed of look directions L24, L26, L27, L41, L45, and L47); and the dark region, where there is no strong sign of photons (composed of the apertures that are neither the bright look direction nor the dim look direction lists and that are mostly composed of wedges W0, W1, and W5-W7, which look away from the Sun). Explicitly, the list of dark look directions is L00-L21, L23, L28-L33, L38-L40, L42, L43, and L48-L79, with the sum of the bright, dim, and dark look directions incorporating all 80 apertures. Note that two of the dark look directions (L03 and L10), which are heavily obscured by the EPI-Hi instrument, were furnished with $2.9 \mathrm{~mm}$ thick aluminum covers to avoid admitting poorly characterized levels of low-energy particles or photons into the apertures; thus, these apertures are not subject to photon contamination. The effect of this is visible in Figure 3(v), where the two look directions (in the six o'clock position) show greatly suppressed "blue" intensity levels relative to the nearly isotropic "red" intensity level. Similarly, L52 (in the one o'clock position), which is obscured by a limb sensor but not covered, also shows a suppressed intensity. Although the bright look directions are roughly those most directed at the Sun, an important distinction is that the six apertures closest to the Sun with thicker lightblocking start foils (L23, L30-L33, and L40) are not included in the bright FOV or dim FOV because the thick foils effectively block the scattered light. It should be noted that, due to changes in the instrument configuration (e.g., the aforementioned dust impact on 2019 April 3, or the gain variations that result from adjusting the potential across the MCPs) or the local photon characteristics (e.g., due to changes in the dust distribution or to the location of the photon source), the specific requirements for defining ion- or photon-dominated rates may change as a function of time.

In addition to the division of the FOV, the energy channels are also split into three independent ranges: low energy (channels T030 and T031, 1-4 keV nucleon ${ }^{-1}$ detected energy, based on the TOF measurement, corresponding to incident energies below $\sim 34 \mathrm{keV}$ when $\mathrm{H}$ is assumed), where accidentals dominate during quiet times; medium energy (channels T016-T029, 4-350 keV nucleon ${ }^{-1}$ detected and $\sim 34-370 \mathrm{keV}$ for incident $\mathrm{H}$ ), where foreground ion measurements are the strongest; and

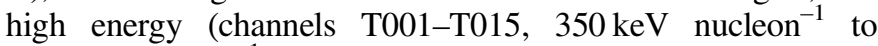
$37 \mathrm{MeV}$ nucleon $^{-1}$ detected and $370 \mathrm{keV}-39 \mathrm{MeV}$ for incident $\mathrm{H}$ ), where cross-talk events dominate during quiet times. Although the upper energy limit is high, no ions were detected above a few hundred $\mathrm{keV}$ nucleon $^{-1}$ (confirmed by EPI-Hi observations). The reason that accidental coincidences are associated with lower-energy intensifications is because randomly distributed start and stop events that accidentally satisfy the TOF logic result in a flat distribution in TOF space, but because of the inverse square relationship between energy and TOF, a large fraction of the longer end of the TOF range corresponds to a small, low-energy range of energies.

This three-by-three grid (Table 2) was used to break up all TOF-only measurements in the [T] and [U] channels (Table 3). There are $5120[\mathrm{~T}]$ and $[\mathrm{U}]$ channels $(80$ apertures $\times 32$ TOFranges $\times 2$ modes) accumulating the individual particle events for which there is no SSD signal (thus the TOF-only designation). The two modes do not operate simultaneously; 
Table 3

PSP/IS $\odot$ IS Data Products Used in This Work ${ }^{\mathrm{a}}$

\begin{tabular}{|c|c|c|c|c|c|c|c|c|c|c|}
\hline $\begin{array}{l}\text { Name of Data } \\
\text { Product }\end{array}$ & $\begin{array}{l}\text { Channel } \\
\text { ID }\end{array}$ & $\begin{array}{l}\text { EPI-Lo } \\
\text { Mode }^{\text {b }}\end{array}$ & ApID & $\begin{array}{c}\text { SSD } \\
\text { Flashing }\end{array}$ & $\begin{array}{c}\text { No. of Energy } \\
\text { Bins Total (Used Here) }\end{array}$ & $\begin{array}{c}\text { Number of } \\
\text { Look Directions }\end{array}$ & $\begin{array}{c}\text { Measured Energy } \\
\text { Range }\left(\mathrm{keV} \text { nucleon }^{-1}\right)\end{array}$ & $\begin{array}{c}\text { Incident Energy } \\
\text { Range }\left(\mathrm{keV} \text { nucleon }^{-1}\right)\end{array}$ & $\begin{array}{l}\text { CDF Data Logical } \\
\text { Name }^{c}\end{array}$ & $\begin{array}{l}\text { CDF Data Variable } \\
\text { Name }^{\mathrm{d}}\end{array}$ \\
\hline Ion TOF & {$[\mathrm{T}]$} & IC & $0 \times 4 b 8$ & none & $32(31)$ & 80 (L00-L79) & $1-47,000$ & $30-47,000$ & psp_isois-epilo_12-ic & $\begin{array}{c}\text { H_Flux_ChanT, } \\
\text { H_CountRate_ChanT }\end{array}$ \\
\hline IC Basic Rate & [ICBR] & IC & $0 \times 4 b 1$ & none & $\mathrm{n} / \mathrm{a}$ & $4(\mathrm{Q} 0-\mathrm{Q} 3)$ & $\mathrm{n} / \mathrm{a}$ & $\mathrm{n} / \mathrm{a}$ & psp_isois-epilo_11-4b1 & $\begin{array}{l}\text { ICB0Qa_Start1CFD, } \\
\text { ICB1Qa_StopCFD, } \\
\text { ICB7M0_InternalPulses }\end{array}$ \\
\hline Particle TOF & {$[\mathrm{U}]$} & $\mathrm{PC}$ & $0 \times 4 c 1$ & $3.2 \mu \mathrm{m} \mathrm{Al}$ & $32(31)$ & 80 (L00-L79) & $1-47,000$ & $30-47,000$ & psp_isois-epilo_12-pc & $\begin{array}{c}\text { H_Flux_ChanU, } \\
\text { H_CountRate_ChanU }\end{array}$ \\
\hline PC Basic Rate & [PCBR] & PC & $0 \mathrm{x} 4 \mathrm{bd}$ & $3.2 \mu \mathrm{m} \mathrm{Al}$ & $\mathrm{n} / \mathrm{a}$ & $4(\mathrm{Q} 0-\mathrm{Q} 3)$ & $\mathrm{n} / \mathrm{a}$ & $\mathrm{n} / \mathrm{a}$ & psp_isois-epilo_11-4bd & $\begin{array}{l}\text { PCB0Qa_Start1CFD, } \\
\text { PCB1Qa_StopCFD, } \\
\text { PCB7M0_InternalPulses }\end{array}$ \\
\hline
\end{tabular}

${ }^{a}$ IS $\odot$ IS data are available at http://spp-isois.sr.unh.edu/data_public/, as well as the NASA Space Physics Data Facility.

${ }^{\mathrm{b}} \mathrm{IC}=$ Ion Composition; PC = Particle Composition.

${ }^{\mathrm{c}}$ The calibration from instrument to physical units is occasionally updated, and some or all of the data set is reprocessed, so users should make sure to use the most recently processed data.

${ }^{\mathrm{d}} Q a$ can take the values $Q 0, Q 1, Q 2$, or $Q 3$, representing the four quadrants. 


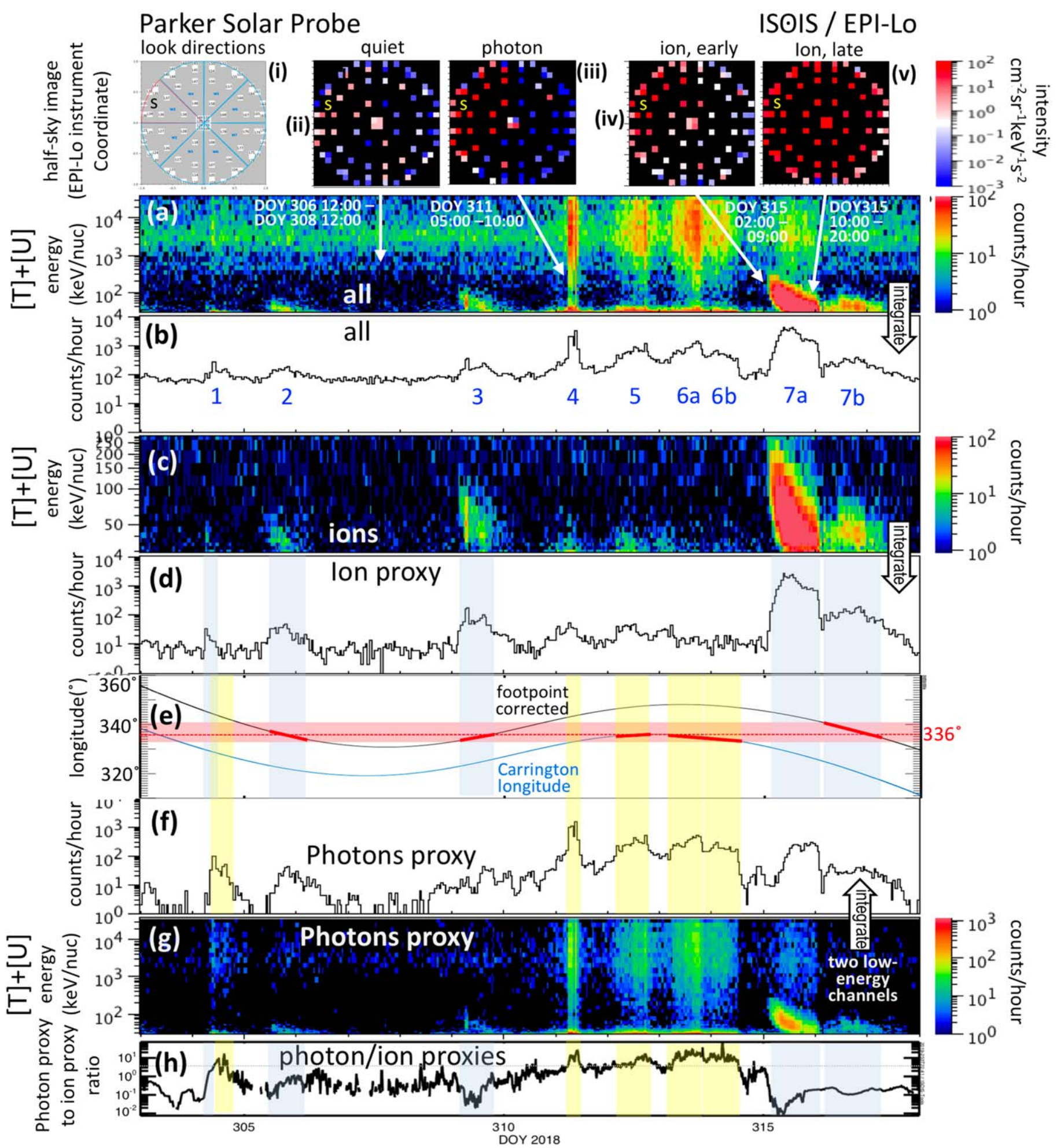

Figure 3. EPI-Lo TOF-only (channels $[\mathrm{T}]$ and $[\mathrm{U}]$ ) measurements and identification of ion and photon activity during the first PSP perihelion pass. The active periods are shown and defined in Table 4. The pairs of spectrograms and energy-integrated rate curves, panels (a) and (b), panels (c) and (d), and panels (g) and (f), show unfiltered data, data composed of dark look directions (at medium and low energies) plus dim look directions (medium energies), and data composed only of bright look directions, respectively. For the case of the photon proxy (panel (f)), only the lowest two energy bins are included in the integrated rate. Panel (h) shows the ratio of the photon to ion proxies, with the division at $\sim 3$ roughly demarking the minimum value for an event to be considered photon dominated, if an event is underway. Panel (e), as described in the text, shows the ion and photon events mapped to their HG longitudes. The $336^{\circ}$ Carrington longitude has two ion events associated with it. Remote observations and data-driven modeling (Figure 7) show indications of solar activity at this longitude. Above panel (a) are five images representing the angular response of the instrument, with image (i) a repeat of Figure 2(b) for reference, image (ii) an intensity image during a $48 \mathrm{hr}$ quiet period, image (iii) a $5 \mathrm{hr}$ image dominated by photons, image (iv) an ion-dominated SEP during the early onset period, and image (v) the late stage of the dispersive events. The instrument wedge most aligned with the Sun is marked with the letter S. 
rather, they (along with two energy-only modes, not discussed here) are multiplexed according to the assigned duty cycle and distributed among eight "slots" per second. During each slot interval, either the Ion SSD is live and the Particle SSD is latent (IC mode) or vice versa (PC mode). This means that lowenergy particles that are incident on the area corresponding to the live SSD but are unable to penetrate the stop foil and the SSD dead layer will be counted, as will ions (of any energy $<47 \mathrm{MeV}$ nucleon $^{-1}$ ) that strike the stop foil above the latent SSD or a region on the live SSD where there is no active area in either mode (such as above the guard rings surrounding each $\mathrm{SSD})$. We have summed the [T] and [U] channels except where we have explicitly calculated the accidental coincidence rate (see Section 3.2). Lacking SSD measurement of energy, the TOF-only measurements do not provide a direct measurement of elemental composition, but by using the TOF versus energy measurements (which do provide composition, with some energy overlap) and requiring consistency with the TOF-only data, some estimate of the composition in the [T] and [U] channels can be made. In this article, we consider only total ions with measured energy $\left(\mathrm{keV}\right.$ nucleon $\left.{ }^{-1}\right)$ and the angular distribution determined from the 80 look directions; see Mitchell et al. (2020) and Desai et al. (2020) for observations using the mass separation capabilities of EPI-Lo. Note that even the energy per nucleon measurement is approximate because of the mass-dependent energy loss in the collimator foils and start foils.

To define the proxy for ion detection, we chose the look directions and energy ranges that were the least impacted by photons; thus, we used the sum of the dark look directions at medium energy, dark look directions at low energy, and dim look directions at medium energy. Table 2 shows this selection highlighted with braces. Besides a roughly constant GCR start and stop accidental signal and GCR cross-talk signal, this ion proxy definition is characterized by ion-dominated foreground (ion start and stop) and ion-dominated background (ion and GCR accidentals and ion cross talk) and not dominated by the photon signal. The ion proxy spectra and summed rate are shown in Figures 3(c) and (d). When compared to the full, unfiltered spectrogram (Figure 3(a)), it can be seen how the dispersive ion events (for which high energies arrive before lower energies) stand out relative to the suppressed photondominated background. There still appears to be some photon contamination even in the dark look directions, as seen at low energies during events E4, E5, and E6a (Figure 3(c)). This is possibly the result of photons scattering or backscattering off zodiacal dust (the $\mathrm{F}$ Corona) or reflecting surfaces on the spacecraft, such as the solar arrays. At the medium energies where most of the ion signal resides, the photon background is largely removed; for example, during the E5 and E6a photon events, at medium energies and all look directions there are $\sim 100$ counts $\mathrm{hr}^{-1}$ at the peaks for all $\mathrm{T}$ channels and $\sim 10$ counts $\mathrm{hr}^{-1}$ in between peaks, while at medium energies and only dark look directions there is a flat signal with fewer than 10 counts hr ${ }^{-1}$ throughout (data not shown). The strong ion event E7a, for all look directions, peaks at $\sim 1650$ counts $\mathrm{hr}^{-1}$ in the medium-energy range versus $\sim 950$ counts $\mathrm{hr}^{-1}$ in the dark look directions. Hence, this selection criterion reduces photon signal by a factor of 10 and the foreground ion signal by less than a factor of 2. Figure 3(a), images (iv) and (v) show the angular distribution during the early and later period of the ion event, the time-varying anisotropy being distinct from the photon signal (image (iii)), which is bright only in the sunward direction.

For the photon proxy, we used the look directions and energies dominated by photons, with the least influence of ions, which are the bright look directions at low energies. Table 2 shows this choice highlighted in brackets. It includes strong photon detections (modality G) and a constant GCR start and stop accidental signal (modality $t$ ). However, there are also nonignorable, ion-dependent contributors to this photon proxy, namely, accidental coincidence between ions and GCRs (modalities e and $\mathbf{p}$, which would be expected at low energies) and, during sizable ion events, the accidental coincidences from uncorrelated foreground starts and stops (modality $u$ ). The two lowest-energy channels (T030-T031 and U030-U031) alone are used to construct the photon proxy rate, shown in Figure 3(f), and it is clear that although there is an ion response, the level is much lower than the photon response. This relative difference in rate change due to ions and photons in either the ion or photon proxy rates is addressed by calculating the ratio of the photon to ion proxy rates as a means to discriminate between photons and ions. The discrimination ratio of the photon proxy rate to the ion proxy rate is used to determine whether the EPI-Lo counts are photon or ion dominated (Figure 3(h)). When the ratio is greater than $\sim 3$ (maxing out at $\sim 50$ during our period of interest) and simultaneously there are intensity enhancements in the overall count rate (Figure 3(b)), the presence of a strong photon signal is indicated. Conversely, when the ratio is below 1 (and as low as 0.01), it is evidence that an ion event transpired. The intermediate $\sim 1-3$ range can be a period of transition or an overlap of particle and photon activity, as during period E1. This soft cutoff at $\sim 3$ is determined empirically; see quantitative accidental coincidence calculations below (Section 3.2).

\subsection{Accidental Coincidence Calculations}

To automate the background removal, we first analyze the foreground and background in TOF-only measurements. The basic rates used here are the start rate $R_{\mathrm{st}}$, stop rate, $R_{\mathrm{sp}}$ (Figure 4(a)), and pulser rate $R_{\mathrm{P}}$ (as reported by the instrument, which is less than or equal to $1 \mathrm{kHz}$ per quadrant owing to the less than $100 \%$ live time of the instrument, for example, while the FPGA processor is busy with data management). The accidental coincidence rate $R_{\text {acc }}^{\prime}$ for a double coincidence within a timing window of duration $\tau$, where the signal order is prescribed (start before stop, in our case), is $R_{\mathrm{acc}}^{\prime}=R_{\mathrm{st}} R_{\mathrm{sp}} \tau$, as written down by Eckart \& Shonka (1938); the factor of 2 in their first equation, which we lack, results from the fact that their signal order is unconstrained. In the case of EPI-Lo there are a number of complications that do not permit the use of this simple form, as follows:

(1) EPI-Lo has the $1 \mathrm{kHz}$ internal pulser (per quadrant) used for live-time corrections with which external signals (such as starts or stops from photons, foreground ions, or GCRs) can form accidental coincidences. For the entire instrument the maximum pulser rate is thus $R_{\mathrm{P}}=4 \mathrm{kHz}$.

(2) The pulser accidentals are frequently tagged as pulser events when a pulser start has an accidental coincidence with an external stop signal (because the start signal is how the position is encoded). However, an accidental coincidence between an external start signal and the 


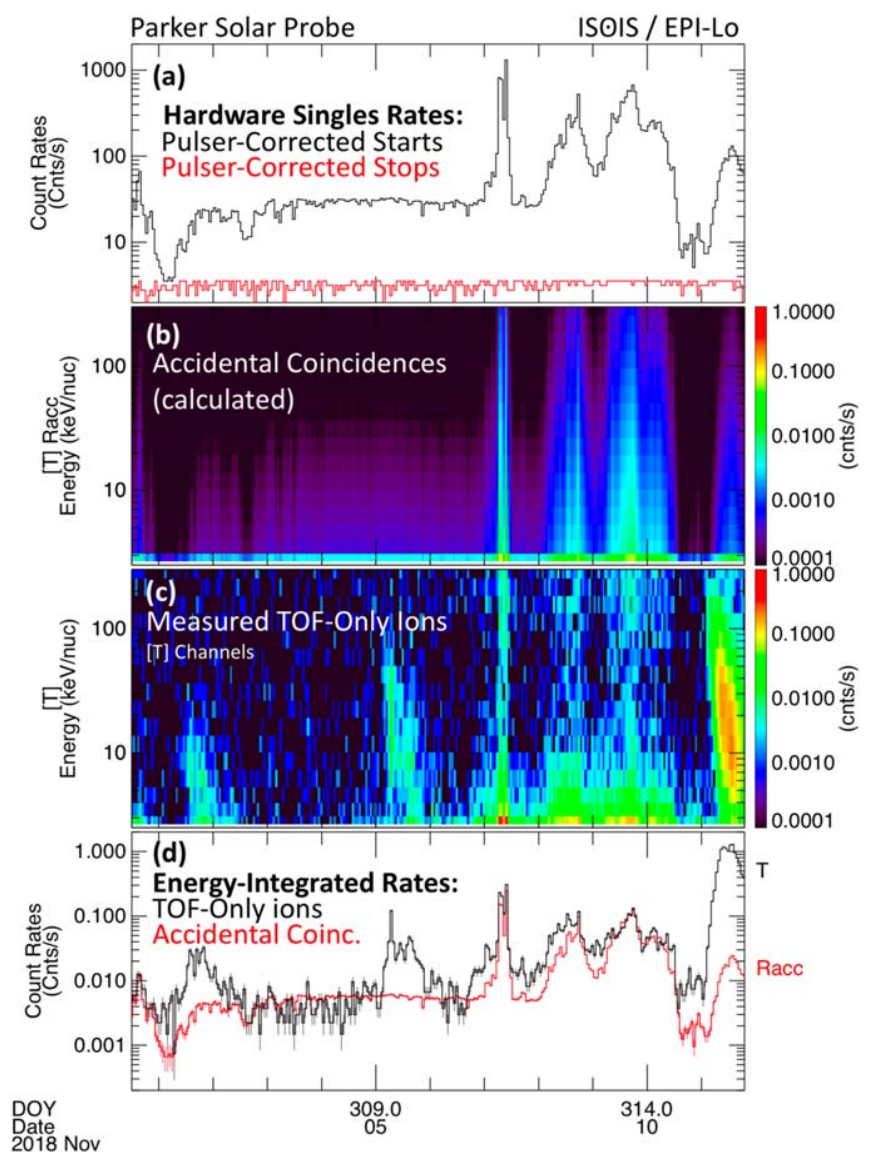

Figure 4. EPI-Lo instrument/engineering rates used to calculate the accidental coincidence rate, an estimate of the photon contribution to the ion measurements. (a) Hardware "singles rates" (i.e., a measure with event logic involving only a single measurement, which requires no coincidence). The start rate (black) for the entire instrument is corrected by subtracting the calibrating internal pulser $(\sim 4000 \mathrm{~Hz})$ and shows a strong response to photon activity, as well as the strongest ion event on DOY 315. The stop rate (red) shows very little variation and is noticeably discretized; this is the result of the lossy compression scheme, which we plan to address in a future flight software update. (b) The accidental coincidences are calculated as described in the text, for each energy channel (with energy- and angular-dependent coincidence windows), binned hourly. The three strong photon events, E4, E5, and E6 (Table 4), are very bright in the measured data (panel (c)) and in the accidental coincidence spectrogram (b). (d) The (red) accidental coincidence and (black) measured ions are energy-integrated forming comparable rates, with statistical errors shown. Unlike Figures 3 and 5, the energies shown here are measured energies, based directly on the TOF measurement, without conversion to incident energies. The magnitude and shape of the three large photon events are captured by the accidental coincidence estimate. The accidental coincidence peak on DOY 315 is about a factor of 50 lower than the observation, indicating that most of the signal is due to a genuine increase in ion counts, with only a small contamination from ion-induced accidental coincidences.

pulser stop will not be tagged as a pulser event (since the stop signal has no position information). This introduces the pulser correction for the start rate $R_{\mathrm{st}}-R_{\mathrm{P}}$ but not the stop rate; thus, the accidental coincidence rate becomes $R_{\mathrm{acc}}^{\prime \prime}=\left(R_{\mathrm{st}}-R_{\mathrm{P}}\right) R_{\mathrm{sp}} \tau$.

(3) This does not quite handle the correction properly because all the rates except the pulser rate have been corrected for the live time. Therefore, the fixed value of $1 \mathrm{kHz}$ should be used rather than the reported pulser rate. But this results in negative start rates during quiet time, so we know that this is an overcorrection. We determined the maximum offset for which no negative net rates resulted, finding $R_{\text {off }}=88 \mathrm{~Hz}$. Thus, $R_{\mathrm{acc}}=\left(R_{\mathrm{st}}-R_{\mathrm{P}}-R_{\mathrm{off}}\right) R_{\mathrm{sp}} \tau$.
(4) Use of this offset is only an approximation, and it reflects the fact that the argument in item (2) neglects the fact that the pulser accidentals (with either start pulser or stop pulser) are not independent accidentals. We must recognize that such accidentals have a reduced probability to occur because of the guaranteed existence of both a start and stop pulser signal. Thus, three cases are likely when an external start signal and a stop form an accidental coincidence. If the external start is before the pulser start, then the accidental will have a nonpulser position and a longer TOF than the pulser setting. If the external start and the pulser start signal are overlapping, then position identification will be skewed by the existence of two pulses; thus, this accidental will have a position that is neither that of the external start nor the position 20 of the pulser. If the external start follows the pulser start, either a normal pulser signal, tagged as such, will result, or multipulse logic will reject the event. Something similar happens with an external stop, except the position will not be shifted, but multipulse logic can veto some such events, meaning that using the stop rate alone will exclude this effect.

(5) Additionally, there is variation in the overall event coincidence window because EPI-Lo utilizes a $10 \mathrm{MHz}$ clock that forces the window to either be $100 \mathrm{~ns}$ or $200 \mathrm{~ns}$, depending on where the signals fall relative to the enforced boundaries separating clock cycles. This effect has not been accounted for and should be most important for TOFs above $100 \mathrm{~ns}$, corresponding to particles having less than $5 \mathrm{keV}$ nucleon ${ }^{-1}$ energy as detected within the instrument.

(6) We wish to determine the accidental rate for each jth energy bin (centered at an energy per nucleon $E_{\mathrm{j}}$ ), so we use the TOF window calculated by taking the difference between the TOF value associated with the lower energy bound and the TOF value associated with the upper energy bound. This, however, must be adjusted by the fact that the effective window is angularly dependent because the instrument has six unique ranges of path lengths (which we will represent for the $i$ th look direction as $\ell_{\mathrm{i}}$ ), so the timing window for a given energy channel is shorter for short path length (high instrument elevation angle) apertures than for long path length apertures. We have accounted for this energy and path-length adjustment $\tau=\tau\left(\ell_{\mathrm{i}}, E_{\mathrm{j}}\right)$, but an implicit assumption was made that the external start and stop signals are uniformly distributed in look direction. This is not the case in general, and an improved estimate will be determined in the future. Integrated over the 80 apertures, we now have the approximate accidental coincidence for the $j$ th energy channel,

$$
R_{\mathrm{acc}, \mathrm{j}}=\sum_{i=L 00}^{L 79}\left(R_{\mathrm{st}, q_{\mathrm{i}}}-\frac{1}{4} R_{\mathrm{P}}-R_{\mathrm{off}}\right) R_{\mathrm{sp}, q_{\mathrm{i}}} \tau\left(\ell_{\mathrm{i}}, E_{j}\right),
$$

where the $q_{\mathrm{i}}$ index indicates that the rates depend on the quadrant: the look directions are associated with the quadrants as follows: $q_{\mathrm{L} 00-\mathrm{L} 19}, q_{\mathrm{L} 20-\mathrm{L} 39}, q_{\mathrm{L} 40-\mathrm{L} 59}$, and $q_{\mathrm{L} 60-\mathrm{L} 79}$ with $Q 0, Q 1, Q 2$, and $Q 3$, respectively.

(7) Finally, we have a lack of precision in the start and stop singles rates that arises from a lossy compression scheme that, when combined with the constant $1 \mathrm{kHz}$ pulser rate and a given integration time, can result in an error up to 
Table 4

Event Definition and Parameters

\begin{tabular}{|c|c|c|c|c|c|c|}
\hline Active Periods & Start Date $\left(\mathrm{DOY}^{\mathrm{a}}\right)$ Time $^{\mathrm{b}}$ & End $\left(\mathrm{DOY}^{\mathrm{a}}\right) \mathrm{Time}^{\mathrm{b}}$ & Type $^{\text {c }}$ & Injection Time & Path Length $^{\mathrm{d}}$ & $P S P-$ Sun Distance \\
\hline E1a & 2018 Oct 31 (304) 06:00 & (304) 08:00 & Ion & Undetermined & Undetermined & $0.25 \mathrm{au}$ \\
\hline E1b & 2018 Oct 31 (304) 08:00 & (304) 11:00 & Photon & $\ldots$ & $\ldots$ & $0.25 \mathrm{au}$ \\
\hline E2 & 2018 Nov 1 (305) 12:00 & (305) 20:00 & Ion & 04:00-07:30 & $0.25 \mathrm{au}$ & $0.22 \mathrm{au}$ \\
\hline E3 & 2018 Nov 5 (309) 03:00 & (309) 20:00 & Ion & 01:00-01:30 & $0.19 \mathrm{au}$ & $0.17 \mathrm{au}$ \\
\hline E4 & 2018 Nov 7 (311) 05:00 & (311) 10:00 & Photon & $\cdots$ & $\cdots$ & $0.17 \mathrm{au}$ \\
\hline E5 & 2018 Nov 8 (312) 04:00 & (312) 20:00 & Photon & $\cdots$ & $\cdots$ & $0.18 \mathrm{au}$ \\
\hline E6a & 2018 Nov 9 (313) 08:00 & (313) 20:00 & Photon & $\cdots$ & $\cdots$ & $0.20 \mathrm{au}$ \\
\hline E6b & 2018 Nov 10 (314) 00:00 & (314) $11: 30$ & Photon & $\cdots$ & $\cdots$ & $0.22 \mathrm{au}$ \\
\hline $\mathrm{E} 7 \mathrm{a}$ & 2018 Nov 11 (315) 02:00 & (315) 24:00 & Ion & 00:00-00:30 & $0.31 \mathrm{au}$ & $0.24 \mathrm{au}$ \\
\hline $\mathrm{E} 7 \mathrm{~b}$ & 2018 Nov 12 (316) 05:00 & (317) 07:00 & Ion & Nondispersive & $\mathrm{n} / \mathrm{a}$ & $0.26 \mathrm{au}$ \\
\hline
\end{tabular}

Notes.

a Day of year.

b Time is spacecraft event time (UTC).

${ }^{\mathrm{c}}$ Either dominantly foreground (ions) or background dominated by photons.

${ }^{\mathrm{d}}$ Path length for dispersive ion events.

$\sim 1 \mathrm{~Hz}$ (seen most easily in the steady stop rate in Figure 4(a)), which is only relevant for low rates, but notice that the pulser-corrected start rate can be as low as $4 \mathrm{~Hz}$. If we have to employ a pulser correction to the stop rate, due to item (4), then this precision loss could become important owing to the fact that the pulsercorrected stop rate is consistently lower than the start rate.

We have used these provisional considerations to calculate the accidental coincidence rate spectrogram shown in Figure 4(b), as described in Equation (1). This compares favorably to the actual measured data in Figure 4(c), where the three large photon events (as first determined by the proxy methods of Section 3.1) are similar in shape and intensity to the accidental calculation. When summed over energies below $300 \mathrm{keV}$ (to avoid a higher-energy cross-talk signal), the curves in Figure 4(d) obtain, showing a strong association between the observed rate increase during the photon events and the estimated accidental rate, while during the dispersive ion events the accidental estimate is at least an order of magnitude lower than the observed rate. This quantitative analysis, although preliminary, supports the empirical method used to form the ion and photon proxies described in Section 3.1 and displayed in Figure 3.

\section{Discussion}

PSP travels at $95 \mathrm{~km} \mathrm{~s}^{-1}$ at closest approach, during the first three perihelia, resulting in an angular motion exceeding the local corotation speed; this means that in a frame rotating with the Sun, PSP undergoes retrograde motion, covering a $17^{\circ}$ heliographic (HG) longitudinal range three times during the course of an $\sim 11$-day perihelion pass, which permits a unique spatial and temporal study into the location, correlation, and persistence of previously unobservable, very small SEP events. In order to identify the solar sources associated with our SEP enhancements, we compute the magnetic FP of PSP mapped back to the solar surface. To identify the PSP Carrington longitudes during photon activity, we simply use the HG longitude of PSP and ignore the inconsequential $<2$-minute light-travel time. We use an estimate for the FP based on a nominal Parker spiral magnetic field connecting the Sun to the spacecraft when the solar wind speed is $400 \mathrm{~km} \mathrm{~s}^{-1}$. The FP longitudes determine the estimated position of the source region of the ions.
To facilitate discussion of the observations, we have identified seven active periods (10 including subdivisions), which are defined in Table 4. In Figure 3, the EPI-Lo ion measurements and photon background responses are presented. Note that although we show the photon responses in the same format as the ion measurements, the photon responses should not be interpreted as calibrated measurements. We present them in this format because it illustrates the problem this background response produces in trying to interpret the foreground ion measurements. To illustrate this difficulty, three spectrograms are shown, all with $1 \mathrm{hr}$ time binning, native energy bins, and common 1-100 counts color scale. Panel (a) includes all energies and look directions as described in Section 3.1. In panel (c), to highlight the ions and suppress the photon background, only data that meet the requirement described in Section 3.1 (highlighted with braces in Table 2) are included. Panel (g) shows only the bright look directions, i.e., those with a large photon response. The three count rates are related to the spectrograms as follows. The panel (b) rate is the spectrogram data in panel (a) integrated over all energies. The panel (d) rate is the spectrogram data in panel (c) integrated over all energies shown in that spectrogram. The rate in panel (f), unlike the other cases, only includes a sum of the two lowest-energy channels in the panel $(\mathrm{g})$ spectrogram-this is because these counts were the most dominated by the photon background and least affected by the ion foreground signal. We note that EPI-Hi detected no intensity enhancements above $1 \mathrm{MeV}$, indicating that these small SEPs are strictly low-energy phenomena.

An important factor in determining the solar source is the derived particle injection time at the Sun. These times can be estimated in two ways: by (1) computing the travel time along the Parker spiral for the highest-energy ions (that arrive at PSP promptly) and (2) fitting the ion dispersion to obtain the injection time and path length. For method (1) the path length is set by the location of PSP and the injection time results from the travel time of the fastest particles observed, and for method (2) the path length and the injection times are constrained by the slope and temporal alignment with the onset envelope present in the spectrogram. Figure 5 shows the ion dispersion by plotting the inverse of velocity versus time of arrival. Both methods of estimating the injection time were in good agreement for the 2018 November 11 (DOY 315) event, given that the dispersive ion increase is well above background. The 

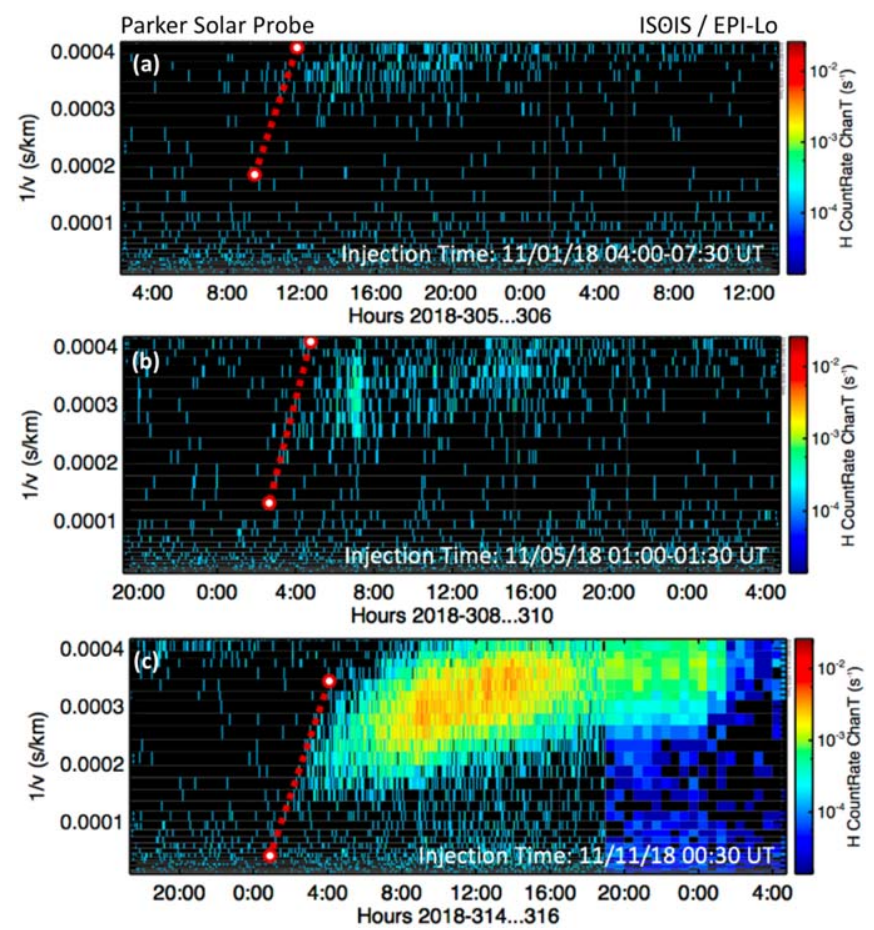

Figure 5. Analysis of dispersive ion events for three of the active periods (defined in Table 4): (a) E2 2018 November 1, (b) E3 2018 November 5, and (c) E7a 2018 November 11. We conducted velocity dispersion analysis to determine the injection times to attempt to identify injection sites. A linear fit in $1 / v$ vs. $t$ space (assuming a stationary source) returns the onset time $t_{\mathrm{o}}$ and path length $L$ of the dispersive event, i.e., $1 / v=\left(t-t_{\mathrm{o}}\right) / L$. Additionally, we determined the travel time on the basis of the timing of the arrival of the highest-energy particles and the distance between the spacecraft and the Sun's surface.

injection times for the 2018 November 1 (DOY 305) and 2018 November 5 (DOY 309) events are more challenging to estimate by fitting the ion dispersion because of the poor statistics. For these events, we assume a path length that was estimated on the basis of scatter-free transport along the nominal Parker spiral (0.19 au for 2018 November 5, DOY 309 and 0.25 au for 2018 November 1, DOY 305). This resulted in an injection time estimate for 2018 November 5 that is in good agreement with travel time calculations and an injection time estimate for 2018 November 1 (DOY 305) that differs from calculations by less than $4 \mathrm{hr}$. These calculated injection times provide a window for searching for possible solar sources.

To determine whether the sites we identified had any corresponding features on the Sun, we consulted remote images from the SECCHI/Extreme Ultraviolet Imager (EUVI; Howard et al. 2008) on STEREO-A (Kaiser et al. 2008) (Figure 6), Wang-Sheeley-Arge (WSA) coronal models (Figure 7(a)), and the Solar Dynamics Observatory's Atmospheric Imaging Assembly (SDO/AIA; Pesnell et al. 2012; Figure 7(b)). We identified a candidate site that is well aligned with the $\sim 336^{\circ}$ longitude determined by EPI-Lo.

The WSA coronal magnetic field model is constructed from an input synoptic map of the photospheric magnetic flux. Solar wind speeds are then estimated at the outer boundary of the model based on an empirical relationship between the wind speed, the flux tube expansion factor, and the distance from the FP of a given magnetic field line to the closest coronal hole boundary (Arge \& Pizzo 2000; Arge et al. 2003, 2004). The model results shown in Figure 7 are based on an Air Force Data
Assimilative Photospheric Flux Transport (ADAPT) photospheric synoptic map. ADAPT assimilates a series of full-disk (Earth-facing) photospheric magnetograms taken over the course of a Carrington rotation, along with empirical knowledge of the way flux is transported across the solar surface, to assemble a pseudo-synchronic map of photospheric magnetic flux at any given time. The ADAPT maps used for this study are based on full-disk magnetograms from the Global Oscillation Network Group (GONG), a network of Michelson-interferometer-based velocity imagers spread across Earth's surface (Harvey \& The Gong Instrument Team 1995; Liebacher 1999). The ADAPT model produces photospheric maps in 12-member ensembles to account for variation in flux motion due to supergranulation, and the ensemble member and source surface height used to determine the FP locations for this study were carefully selected to produce a model that best matched the observed solar wind speed and interplanetary magnetic field polarity observed by PSP. For a complete description of both WSA and ADAPT and the parameters specific to the model output discussed in this paper, see Szabo et al. (2020).

From 2018 November 1 (DOY 305), the PSP FPs are located near the boundary of a small midlatitude coronal hole toward the western hemisphere but then shift to a coronal hole closer to central meridian by 2018 November 11 (DOY 315). Figure 7(b) shows magnetic field extrapolations based on a global, time-dependent magnetohydrodynamic (MHD) model, the MHD Algorithm outside a Sphere (MAS; Mikić et al. 2018; Riley et al. 2019). The simulations are based on the corresponding Carrington rotation for the event using photospheric magnetic field synoptic maps from SDO's Helioseismic and Magnetic Imager (HMI; Scherrer et al. 2012). The magnetic field lines are overlaid on SDO/AIA Fe XII $193 \AA$ EUV emission corresponding to corona/flare plasma. Openfield flux associated with these two coronal holes provides a possible route for the escape of accelerated ions.

We embarked on a thorough search for low coronal activity associated with these events, which should have been straightforward given the relative corotation of PSP relative to the Sun and the minimum of solar activity. Although we were unable to find unambiguous counterparts to the particle events, we identified several candidates:

(1) For E2, we found a mini-CME (Podladchikova et al. 2010) in AIA 193 $\AA$ images on 2018 November 1 4:16 UT from a region at Carrington coordinates $329^{\circ},-16^{\circ}$ (Figure 104(a)). The event lasts only 10-15 minutes, though. A small filament is also ejected at around 3:45 from $84 \mathrm{~W}$ (corresponding to CL22), which is $54^{\circ}$ west of PSP and may be magnetically connected to the spacecraft. This location is interesting because it seems to be connected to a slow-rising CME detected by WISPR (Vourlidas et al. 2016) with a source region extending further behind the limb (Hess et al. 2020).

(2) For E3, the potential source region is beyond the limbs of either AIA or EUVI-A, so we cannot identify a source location. We note, however, that WISPR and other coronagraphs detected a CME on that day. The ensuing 3D reconstruction (Wood et al. 2020) suggests a source location at CL339, which is very close to the PSP estimated magnetic FP (CL335). It is therefore likely that the particles are associated with the CME. 

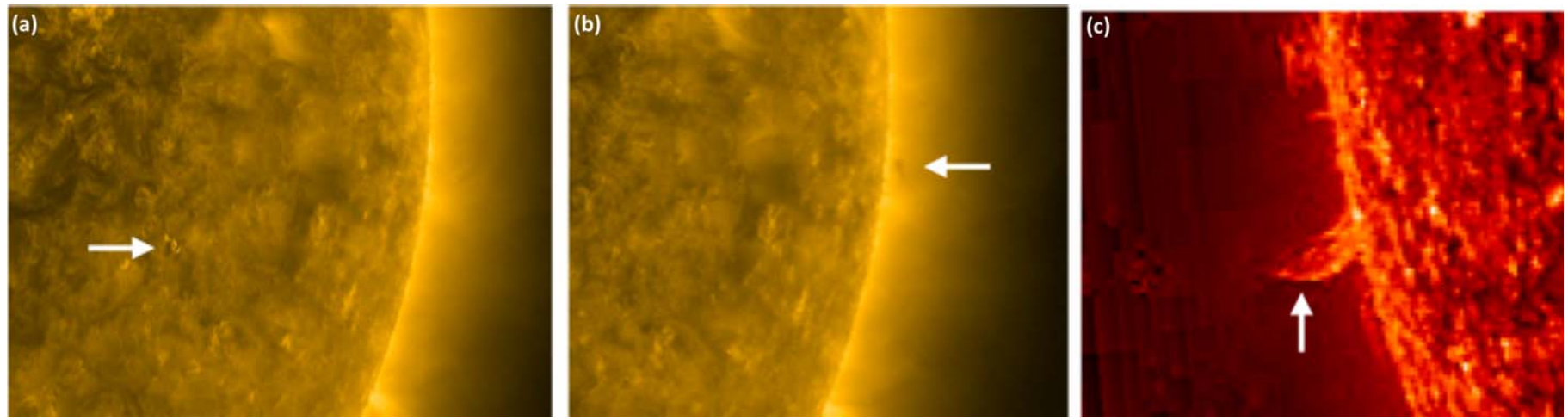

Figure 6. Candidate solar sources of events E2 and E7a. On 2018 November 1 AIA $193 \AA ̊$ images show (a) a mini-CME at 3:45 UT and (b) a small filament ejection at 4:16 UT as possible sources for event E2. (c) EUVI-A $304 \AA$ images on 2018 November 11 at 22:41 UT showing a surge that may be associated with event E7a.
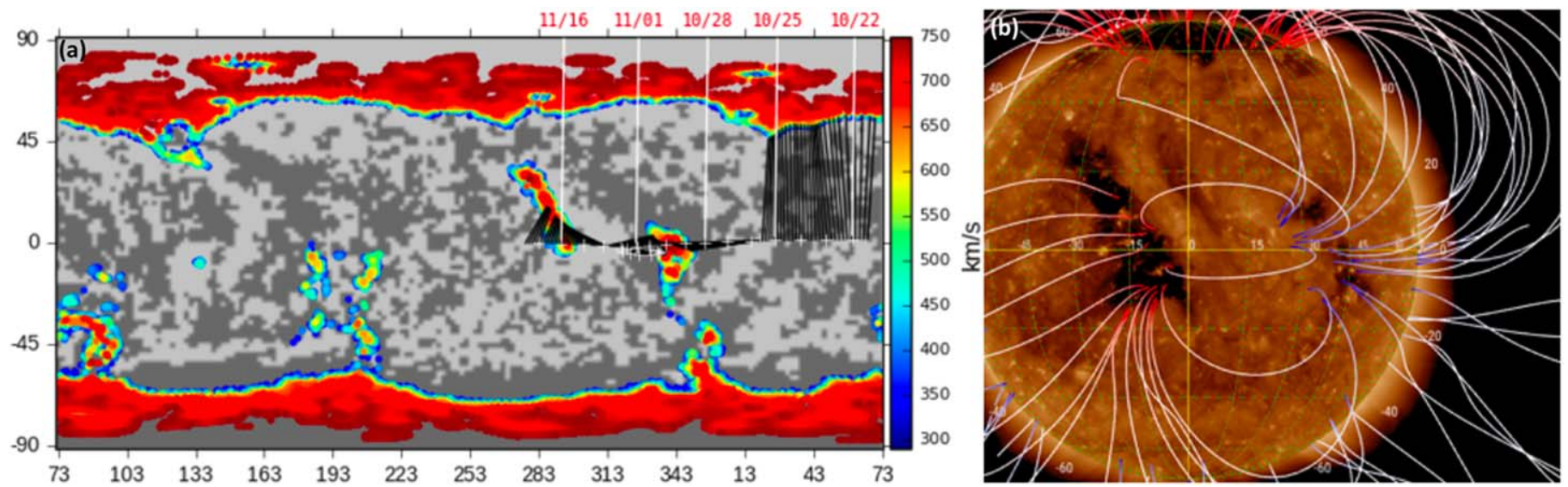

Figure 7. (a) ADAPT-WSA-derived coronal holes at $1 R_{s}$ with model-derived solar wind speed in color scale and $P S P$ connectivity to the solar surface during the first solar encounter of Parker Solar Probe. The last GONG observations assimilated into the ADAPT input map were from 2018 November 4 00:00:00 UTC at approximately $253^{\circ}$ Carrington longitude (CR 2210). The field polarity at the photosphere is indicated by the light/dark (positive/negative) gray contours. White tick marks represent $P S P$ 's location mapped back to 5 solar radii with dates labeled above in red. Black lines show connectivity between spacecraft and solar wind source region at 1 solar radius. (b) SDO's AIA 193A solar disk showing two coronal holes associated with the FPs of PSP.

(3) For E7a, the only ejective activity is a surge in EUVI-A $304 \AA$ Amages on 2018 November 10 at 22:33-22:58 UT, $2 \mathrm{hr}$ earlier than the estimated injection time (Table 4). It occurs at Carrington coordinates $330^{\circ},-15^{\circ}$, which again are very close to the $P S P$ subsolar point. The activity may be associated with a very slow CME ejection that was intercepted by PSP after 20:00 UT on 2018 November 11 (McComas et al. 2019; Giacalone et al. 2020).

(4) For E7b, we are unable to find any indications for ejections before the onset of the event in PSP at 7UT. We note, however, that PSP was crossing the CME mentioned earlier, suggesting that these particles were likely accelerated closer to the Sun but largely remained trapped within the mostly closed CME structure passing the spacecraft; this spatial transition would explain the nondispersive character of this event. The largely bidirectional nature of $314 \mathrm{eV}$ electron pitch-angle distributions measured by SWEAP during event E7b (Kasper et al. 2019) supports the contention that the passing structure was predominantly closed.

Overall, we cannot make strong statements about the solar origins of the four events. But there are consistencies in the tentative identifications that allow us to provide an interpretation. The inspection of the solar images suggests that the events may be originating from the same longitude (around CL335), close to the equatorial coronal hole that $P S P$ was connected to throughout the perihelion. They also seem to be related to the source region of the three CMEs detected by WISPR and the other coronagraphs (i.e., CMEs on November 1, 5, and 12). These events seem to be successive instances of a long-duration streamer blowout (Vourlidas \& Webb 2018). This indicates the reconfiguration of the global magnetic field, possibly due to the evolution of the equatorial coronal hole (which appeared on disk around 2018 October 30). In other words, both the CME and particle activity are manifestations of this "global reconnection" event. The low energies of both the CMEs and the particle events are consistent with the slow evolution of the blowout.

\section{Summary}

EPI-Lo measures ion intensity, energy, composition, and anisotropy, by design, and also responds to photons that are most likely scattered by zodiacal dust. Utilizing thicker start foils to suppress photon contamination unfortunately degrades low-energy ion response, so this mitigation was used sparingly; consequently, there are several generally sunward apertures with thin start foils where photons produce backgrounds, mostly through accidental coincidence of uncorrelated 
transmitted photon start and stop triggers. The result is that EPI-Lo responds to SEPs and solar photons, and through directional and energy filtering as well as accidental coincidence calculations, the ion signal can be largely separated from the photon backgrounds, as we have detailed. By exploiting a retrograde orbital configuration, permitting repeated observation of a small range of heliographic longitudes over an extended time $\left(17^{\circ}\right.$ over 11 days with three visits at each angular position), we have been able to study the relationship between SEPs and coronal activity where these events might originate. Fortuitously, three of the SEP events show clear dispersion that we used to compute injection times at the Sun. Inspection of coronal activity near the derived magnetic FP for PSP revealed potential associations with CMEs. While these associations are tentative, we note that PSP has detected extremely small, low-energy SEP events that are not detectable at 1 au and offer a new perspective on particle acceleration. Future perihelion approaches of PSP coordinated with remote observations at 1 au will provide new opportunities to study these SEPs (and potentially solar photon enhancements) in detail. Such opportunities to better identify the solar sources, particularly coordinated with remote observations at 1 au and with STEREO-A, will occur during the following PSP perihelia: 2020 January 29, 2020 June 7, 2021 April 29, 2022 February 25, and 2023 March 17.

This work was supported by NASA's Parker Solar Probe mission, contract NNN06AA01C. We are indebted to the exceptional, dedicated efforts of those who have made this mission and this experiment possible, in particular the engineers, scientist, and administrators who designed and built EPI-Lo. P. Kollmann, J. Peachy, and J. Vandegriff (at JHU/APL) are owed special thanks for their contribution to the scientific configuration and instrumental analysis of EPI-Lo. STEREO work is supported by NASA grant 80NSSC19K1261 (A.V.). This work utilizes data produced collaboratively between Air Force Research Laboratory (AFRL) and the National Solar Observatory. The ADAPT model development is supported by AFRL. The IS $\odot$ IS data and visualization tools are available to the community at https:// spacephysics.princeton.edu/missions-instruments/isois; data are also available via the NASA Space Physics Data Facility (https://spdf.gsfc.nasa.gov/).

\section{ORCID iDs}

M. E. Hill @i https://orcid.org/0000-0002-5674-4936

D. G. Mitchell ib https://orcid.org/0000-0003-1960-2119

R. C. Allen (1) https://orcid.org/0000-0003-2079-5683

G. A. de Nolfo (1) https://orcid.org/0000-0002-3677-074X

A. Vourlidas (10 https://orcid.org/0000-0002-8164-5948

S. I. Jones (1) https://orcid.org/0000-0001-9498-460X

D. J. McComas (10) https://orcid.org/0000-0001-6160-1158

J. G. Mitchell (i) https://orcid.org/0000-0003-4501-5452

J. R. Szalay (i) https://orcid.org/0000-0003-2685-9801

S. Wallace (i) https://orcid.org/0000-0002-1091-4688

E. R. Christian (1) https://orcid.org/0000-0003-2134-3937

C. M. S. Cohen (1) https://orcid.org/0000-0002-0978-8127

A. B. Crew (1) https://orcid.org/0000-0002-7493-2682

M. I. Desai (1) https://orcid.org/0000-0002-7318-6008

C. J. Henney (1) https://orcid.org/0000-0002-6038-6369

C. J. Joyce (1) https://orcid.org/0000-0002-3841-5020

S. M. Krimigis (1) https://orcid.org/0000-0003-2781-2386

R. A. Leske (1) https://orcid.org/0000-0002-0156-2414
R. A. Mewaldt (i) https://orcid.org/0000-0003-2178-9111

K. S. Nelson (1) https://orcid.org/0000-0003-3807-4518

E. C. Roelof (i) https://orcid.org/0000-0002-2270-0652

N. A. Schwadron (1) https://orcid.org/0000-0002-3737-9283

\section{References}

Arge, C. N., Luhmann, J. G., Odstrcil, D., Schrijver, C. J., \& Li, Y. 2004, JASTP, 66, 1295

Arge, C. N., Odstrcil, D., Pizzo, V. J., \& Mayer, L. R. 2003, in AIP Conf. Proc. 679, SOLAR WIND TEN, ed. M. Velli et al. (Melville, NY: AIP), 190

Arge, C. N., \& Pizzo, V. J. 2000, JGR, 105, 10465

Bale, S., Badman, S. T., Bonnell, J. W., et al. 2019, Natur, 576, 237

Battaglia, M., \& Benz, A. O. 2006, A\&A, 456, 751

Benz, A. O. 2008, LRSP, 5, 1

Bučík, R., Wiedenbeck, M. E., Mason, G. M., et al. 2018, ApJL, 869, L21

Cane, H. V., von Rosenvinge, T. T., Cohen, C. M. S., \& Mewaldt, R. A. 2003, GeoRL, 30, 8017

Cohen, C. M. S., Christian, E. R., Cummings, A. C., et al. 2020, ApJS, doi:10. 3847/1538-4365/ab4c38

Cohen, C. M. S., Mason, G. M., Mewalt, R. A., et al. 2008, in AIP Conf. Proc. 1039, Particle Acceleration and Transport in the Heliosphere and Beyond, ed. G. Li et al. (Melville, NY: AIP), 118

Desai, M. I., Mitchell, D. G., Szalay, J. R., et al. 2020, ApJS, doi:10.3847/ $1538-4365 / \mathrm{ab} 65 \mathrm{ef}$

Eckart, C., \& Shonka, F. R. 1938, PhRv, 53, 752

Fleishman, G. D., Kontar, E. P., Nita, G. M., \& Gary, D. E. 2011, ApJL, 731, L19

Fox, N. J., Velli, M. C., Bale, S. D., et al. 2014, SSRv, 204, 7

Giacalone, J., Mitchell, D. G., Allen, R. C., et al. 2020, ApJS, doi:10.3847/ $1538-4365 / a b 5221$

Harvey, J. \& The GONG Instrument Team 1995, in ASP Conf. Ser. 76, GONG '94: Helio- and Astero-Seismology from the Earth and Space, ed. R. K. Ulrich et al. (San Francisco, CA: ASP), 432

Hess, P., Rouillard, A. P., Kouloumvakos, A., et al. 2020, ApJS, doi:10.3847/ 1538-4365/ab4ffo

Hill, M. E., Mitchell, D. G., Andrews, G. B., et al. 2017, JGRA, 122, 1513

Howard, R. A., Moses, J. D., Vourlidas, A., et al. 2008, SSRv, 136, 67

Howard, R. A., Vourlidas, A., Bothmer, B., et al. 2019, Natur, 576, 232

Joyce, C. J., McComas, D. J., Christian, E. R., et al. 2020, ApJS, doi:10.3847/ $1538-4365 / \mathrm{ab} 5948$

Kaiser, M. L., Kucera, T. A., Davila, J. M., et al. 2008, SSRv, 136, 5

Kasper, J. C., Bale, S. D., Belcher, J. W., et al. 2019, Natur, 576, 228

Kinnison, J., Lockwood, M. K., Fox, N., Conde, R., \& Driesman, A. 2013, in in Proc. IEEE Aerospace Conf., Solar Probe Plus: A Mission to Touch the Sun (Piscataway, NJ: IEEE), 144

Krucker, S., Battaglia, M., Cargill, P. J., et al. 2008, A\&ARv, 16, 155

Krucker, S., Hudson, H. S., Glesener, L., et al. 2010, ApJ, 714, 1108

Leske, R. A., Christian, E. R., Cohen, C. M. S., et al. 2020, ApJS, doi:10.3847/ $1538-4365 / \mathrm{ab} 5712$

Li, C., Tang, Y. H., Dai, Y., Fang, C., \& Vial, J.-C. 2007a, A\&A, 472, 283

Li, C., Tang, Y. H., Dai, Y., Zong, W. G., \& Fang, C. 2007b, A\&A, 461, 1115

Liebacher, J. W. 1999, AdSpR, 24, 173

Lin, R. P., Krucker, S., Hurford, G. J., et al. 2003, ApJL, 595, L69

Masuda, S., Kosugi, T., Hara, H., Tsuneta, S., \& Ogawara, Y. 1994, Natur, 371,495

McComas, Christian, E. R., Cohen, C. M. S., et al. 2019, Natur, 576, 223

McComas, D. J., Alexander, N., Angold, N., et al. 2016, SSRv, 204, 187

Mikić, Z., Downs, C., Linker, J. A., et al. 2018, NatAs, 2, 913

Mitchell, D. G., Giacalone, J., Allen, R. C., et al. 2020, ApJS, doi:10.3847/ $1538-4365 / \mathrm{ab} 63 \mathrm{cc}$

Nitta, N. V., Mason, G. M., Wang, L., Cohen, C. M. S., \& Wiedenbeck, M. E. 2015, ApJ, 806, 235

Pesnell, W. D., Thompson, B. J., \& Chamberlin, P. C. 2012, SoPh, 275, 3

Podladchikova, O., Vourlidas, A., Van der Linden, R. A. M., Wülser, J.-P., \& Patsourakos, S. 2010, ApJ, 709, 369

Ramaty, R., Mandzhavidze, N., Kozlovsky, B., \& Murphy, R. J. 1995, ApJL, 455, L193

Ramaty, R. K., \& Mandzhavidze, N. 2000, in IAU Symp. 195, Highly Energetic Physical Processes and Mechanisms for Emission from Astrophysical Plasmas, ed. P. C. H. Martens, S. Tsuruta, \& M. A. Weber (San Francisco, CA: ASP), 123

Reames, D. V. 1999, SSrv, 90, 413

Riley, P., Down, C., Linker, J. A., et al. 2019, ApJL, 784, L15 
Scherrer, P. H., Schou, J., Bush, R. I., et al. 2012, SoPh, 275, 207

Schwadron, N. A., Bale, S., Bonnell, J. W., et al. 2020, ApJS, doi:10.3847/ $1538-4365 /$ ab5527

Shibatta, K., Nozawa, S., \& Matsumoto, R. 1992, PASJ, 44, 265

Shimojo, M., Hashimoto, S., Shibata, K., et al. 1996, PASJ, 48, 123

Shimojo, M., Shibata, K., \& Harvey, K. L. 1998, SoPh, 178, 379

Szabo, A., Larson, D., Whittlesey, P., et al. 2020, ApJS, doi:10.3847/1538-4365/ ab5dac
Szalay, J. R., Pokorný, P., Bale, S. D., et al. 2020, ApJS, doi:10.3847/15384365/ab50c1

Vourlidas, A., Howard, R. A., Plunkett, S. P., et al. 2016, SSRv, 204, 83

Vourlidas, A., \& Webb, D. F. 2018, ApJ, 861, 103

Wiedenbeck, M. E., Bučík, R., Mason, G. M., et al. 2020, ApJS, doi:10.3847/ $1538-4365 /$ ab5963

Wood, B. E., Hell, P., Howard, R. A., Stenborg, G., \& Wang, Y.-M. 2020, ApJS, doi:10.3847/1538-4365/ab5219 